\title{
Tuberculosis Among Temporary Visa Holders Working in the Tourism Industry — United States, 2012-2014
}

\author{
Meghan P. Weinberg, PhD ${ }^{1,2}$; Cara Cherry, DVM ${ }^{1,3}$; Julie Lipnitz ${ }^{4}$; Linus Nienstadt, MPH${ }^{5}$; April King-Todd, MPH'; Maryam B. Haddad, MSN7; \\ Michelle Russell, $\mathrm{MPH}^{8}$; David Wong, MD; Peter Davidson, $\mathrm{PhD}^{2}$; Jevon McFadden, MD²,10; Corinne Miller, PhD²
}

Tuberculosis (TB) is a contagious bacterial disease of global concern. During 2013, an estimated nine million incident TB cases occurred worldwide (1). The majority (82\%) were diagnosed in 22 countries, including South Africa and the Philippines, where annual incidence was $860 \mathrm{~TB}$ cases per 100,000 persons and $292 \mathrm{~TB}$ cases per 100,000 persons, respectively (1). The $2013 \mathrm{~TB}$ incidence in the United States was three cases per 100,000 persons (2). Under the Immigration and Nationality Act, TB screening is required for persons seeking permanent residence in the United States (i.e., immigrants and refugees), but it is not routinely required for nonimmigrants who are issued temporary visas for school or work (3). A portion of the U.S. tourism industry relies on temporary visa holders to accommodate seasonal and fluctuating demand for service personnel (4). This report describes three foreign-born persons holding temporary visas who had infectious $\mathrm{TB}$ while working at tourist destinations in the United States during 2012-2014. Multiple factors, including dormitory-style housing, transient work patterns, and diagnostic delays might have contributed to increased opportunity for TB transmission. Clinicians in seasonally driven tourist destinations should be aware of the potential for imported TB disease in foreign-born seasonal workers and promptly report suspected cases to health officials.

\section{Case Reports}

Case 1. In March 2012, a man aged 25 years from the Philippines arrived in Arizona to work as a cafeteria attendant in a National Park Service lodge. The rural county in which the park is located typically reported five TB cases each year. The man resided in an employee cabin with two roommates. $\mathrm{He}$ had been treating himself intermittently with levofloxacin for neck swelling that began in January 2012; in February 2012, he experienced fever, night sweats, and cough. After working in Arizona for 3 months (March-May 2012), he relocated to Minnesota in June to visit family and find other work. Five days after his arrival in Minnesota, he was admitted to a hospital. He received a diagnosis of acid-fast bacilli (AFB) smear-positive pulmonary TB disease and disseminated TB of the neck, lung, liver, and spleen. Cultures grew Mycobacterium tuberculosis that was resistant to isoniazid and levofloxacin, and the genotype was not previously reported in the United States (2). His TB risk factors included previous residence in the Philippines.
During the ensuing TB contact investigation, 10 employees in Arizona were evaluated; 19 additional contacts, including the patient's two roommates, were no longer working at the park and unable to be contacted for a TB evaluation. Among the 10 employees who received a tuberculin skin test (TST), one female had a positive result, but no TB symptoms and a normal chest radiograph; health professionals determined that she probably had latent TB infection before the recent exposure and did not recommend further testing. The remaining nine persons had negative TST results (induration $<5 \mathrm{~mm}$ ) at initial and follow-up testing. In Minnesota, three household contacts were identified, including one foreign-born household contact who had a history of treated latent TB infection, and two persons who had negative TST results. No additional active TB cases were identified among screened contacts, and no genotype-matching cases had been reported in the United States as of March 18, 2016 (5).

Case 2. In April 2012, a man aged 49 years from the Philippines arrived in Michigan for temporary employment at resort A on Mackinac Island, which has a population of approximately 500 persons and had not reported a TB case since 1995. The man worked as a butcher at the resort restaurant and lived in a dormitory with one roommate. When the resort closed for the season in October 2012, he relocated to California. In May 2013, he was admitted to a hospital with cough, weight loss, night sweats, chills, fever, and shortness of breath; he reported that his symptoms had begun while working in Michigan. He received a diagnosis of AFB smear-positive pulmonary TB disease. The $M$. tuberculosis isolate was susceptible to first-line TB medications isoniazid, rifampin, ethambutol, and pyrazinamide. The genotype was well-established in other parts of the United States (i.e., >100 previous TB cases since 2005), but had not been seen before in Michigan. His TB risk factors included diabetes and previous residence in the Philippines.

A contact investigation was initiated on Mackinac Island during the 2013 tourist season. Thirty-six (53\%) of 68 employees who had had contact with the index patient during 2012 had left the state and did not return; health authorities in the jurisdictions to which they traveled were notified. The remaining $32(47 \%)$ employees had returned to the island and were evaluated for TB. Nineteen (59\%) had either a negative TST 
or interferon-gamma release assay (IGRA) result (G). The 13 (41\%) persons with positive IGRA results were all temporary employees from the Philippines; none had a chest radiograph consistent with active disease and all were considered to have latent TB infection. In California, five family members of the patient were contacts: one had a history of treated latent TB infection, and one of the remaining four had a positive IGRA result and was considered to have latent TB infection. No additional active TB cases were identified among screened contacts. In 2014, a genotype-matching TB case was diagnosed in another Filipino immigrant in Michigan; no epidemiologic association between the two patients is evident.

Case 3. In April 2014, a woman aged 21 years from South Africa arrived for temporary employment at resort B on Mackinac Island. She worked as a housekeeper and laundry attendant and lived in a dormitory with three roommates. In June-July 2014, she sought medical care five times at both a local emergency department and a clinic, where she reported worsening signs and symptoms of pneumonia that included shortness of breath, cough, and weight loss. A different physician examined the patient at each visit. In August 2014, she received a diagnosis of AFB smear-positive pulmonary TB disease. The M. tuberculosis isolate was resistant to isoniazid and the genotype was not previously reported in the United States. Her TB risk factors included contact in December 2013 with a relative with active TB disease, and previous residence in South Africa.

IGRAs were performed on all 26 resort employees who had contact with the index patient. Fourteen $(54 \%)$ had positive IGRA results, including 11 temporary employees from South Africa, two U.S.-born year-round employees, and one Jamaicaborn seasonal employee. None had a history of known TB infection and all were considered to have latent TB infection. One U.S.-born contact who initially tested negative by IGRA had a positive IGRA result at the 8-week follow-up examination, providing evidence of recent $\mathrm{TB}$ infection. No additional active TB cases were identified among screened contacts, and no genotype-matching cases had been reported in the United States as of March 18, 2016.

\section{Discussion}

This report documents three incident cases of infectious TB among foreign-born, temporary workers. In addition to vacation resorts and national parks, sectors of the U.S. tourism industry that rely on temporary visa holders to accommodate the fluctuating and seasonal demand for service personnel include amusement parks, ski lodges, and cultural or historical sites (4). Although the cases described here were counted for the purposes of national TB surveillance, TB incidence among temporary visa holders is difficult to estimate, in part because $\mathrm{TB}$ cases are not included in official case counts when a person is in the United States for $<90$ days (2). Despite this exclusion, approximately two thirds of TB cases in the United States occur among foreign-born persons, and their corresponding TB incidence in 2014 ( 15.4 cases per 100,000 population) was $>10$-fold higher than that among U.S.-born persons (1.2 cases per 100,000 population) (2).

TB screening is not routinely required for persons entering the United States as nonimmigrants (3). During 2013, the U.S. Department of State granted temporary admission to approximately 600,000 students and 400,000 temporary workers and their families (7). The length of stay for these students and temporary workers ranged from months to years, depending on visa type $(7,8)$.

This case series was consistent with a 2005-2006 crosssectional study that determined seeking care for TB symptoms to be the primary reason for the TB diagnosis among temporary visa holders (9). Lack of TB awareness among clinicians can contribute to delayed diagnoses. Diagnostic and treatment delays extend the patient's infectious period, thereby allowing increased opportunities for transmission. In the third case report, the patient had sought medical attention five times for worsening signs and symptoms, including weight loss, cough, and shortness of breath, yet $\mathrm{TB}$ remained undiagnosed for 3 months.

$\mathrm{TB}$ contact investigations among temporary workers are also challenging. Tourism industries have substantial turnover in seasonal employment. In two of the case reports described here, the majority of contacts, including roommates at high risk for $\mathrm{TB}$, had left the state or country at the time contact investigations were initiated, and could not be reached. However, secondary TB cases within the United States as a consequence of any of these three cases seem unlikely, given the nationally unique $M$. tuberculosis genotypes for cases 1 and 3, and birth in the Philippines as the only known commonality between case 2 and other TB cases with that genotype.

The findings in this report are subject to at least two limitations. First, because the majority of infected contacts were temporary employees from high TB incidence countries where the contacts might have been previously infected, interpreting TB test results was challenging. A positive TB test does not necessarily mean that transmission occurred as a result of exposure to the TB patients described here. Second, these three recent $\mathrm{TB}$ cases among foreign-born temporary workers might not be representative of all cases; no generalizations can be made regarding all temporary workers.

Increased awareness concerning the potential for active TB among foreign-born temporary workers is needed. Public 


\section{Summary}

What is already known about this topic?

Tuberculosis (TB) is a global disease; the majority of TB cases in the United States occur among foreign-born persons. TB screening requirements exist for persons seeking permanent status in the United States (i.e., immigrants and refugees), but not for temporary visitors (e.g., students and workers).

What is added by this report?

Three foreign-born persons holding temporary visas had infectious TB while working at U.S. tourist destinations. Multiple factors, including dormitory-style housing, transient work patterns, and diagnostic delays might have contributed to increased opportunity for TB transmission.

What are the implications for public health practice?

Public health authorities might consider providing TB education for employers and clinicians in seasonally driven tourist destinations. Employers might consider implementing TB screening for temporary workers from countries with a high incidence of TB cases. All employers should encourage employees to seek medical attention early during the course of an illness. Clinicians should be aware of the potential for imported TB disease in foreign-born seasonal workers and promptly report suspected cases to health officials to limit TB transmission.

health authorities might consider providing $\mathrm{TB}$ education for employers and clinicians in the tourism sector. Employers might consider implementing TB screening for temporary workers from countries with a high incidence of $\mathrm{TB}$ cases, and all employers should encourage employees to seek medical attention early during the course of an illness. Clinicians should promptly recognize TB signs and symptoms and inquire about previous travel to or residence in countries with a high incidence of TB cases.

A medical exam that includes TB screening is required for persons seeking permanent residence in the United States, including immigrants and refugees, and CDC has the U.S. regulatory oversight of the overseas medical examination process (42 CFR, Part 34) (3). As part of the National Action Plan for Combating Antibiotic Resistant Bacteria initiative, CDC is working with interagency partners to expand premigration $\mathrm{TB}$ screening beyond immigrants and refugees (10). Until global TB elimination is reached, increased TB awareness among clinicians serving foreign-born temporary workers, followed by prompt treatment and public health follow-up after active TB is diagnosed, is necessary to reduce the potential for TB transmission.

\section{Acknowledgments}

Marette Gebhardt, Mary Ellen Ormsby, Mare Schumacher, Coconino County Public Health Services District; Nadya Sabuwala, Ann Sittig, Minnesota Department of Health; Nicholas Derusha,
James Terrian, Luce-Mackinac-Alger-Schoolcraft District Health Department; Jim Collins, Jennie Finks, Xiao Qing Wang, Cassandra McNulty, Michigan Department of Health and Human Services; Marie de Perio, National Institute for Occupational Safety and Health, CDC; Danielle Buttke, Wildlife Health Branch, Biological Resources Division and Office of Public Health, National Park Services; Michael Gronostaj, Jennifer Wright, Division of Scientific Education and Professional Development, CDC.

\footnotetext{
${ }^{1}$ Epidemic Intelligence Service, CDC; ${ }^{2}$ Michigan Department of Health and Human Services; ${ }^{3}$ Office of Public Health and Wildlife Health Branch, Natural Resource Stewardship and Science, National Park Service, Fort Collins, Colorado; ${ }^{4}$ Luce-Mackinac-Alger-Schoolcraft District Health Department, St. Ignace, Michigan; ${ }^{5}$ Coconino County Public Health Services District, Flagstaff, Arizona; ${ }^{6}$ Los Angeles County Department of Public Health, California; ${ }^{7}$ Division of Tuberculosis Elimination, National Center for HIV/AIDS, Viral Hepatitis, STD, and TB Prevention, CDC; ${ }^{8}$ Division of Global Migration and Quarantine, National Center for Emerging and Zoonotic Infectious Diseases, CDC; ${ }^{9}$ Office of Public Health, National Park Service, Albuquerque, New Mexico; ${ }^{10}$ Career Epidemiology Field Officer Program, CDC.

Corresponding authors: Meghan P. Weinberg, MPWeinberg@cdc.gov, 517-241-4054; Cara Cherry, CCherry@cdc.gov, 970-267-7230.
}

\section{References}

1. World Health Organization. Global tuberculosis report 2014. Geneva, Switzerland: World Health Organization; 2014. http://apps.who.int/ iris/bitstream/10665/137094/1/9789241564809_eng.pdf

2. CDC. Reported tuberculosis in the United States, 2014. Atlanta, GA: US Department of Health and Human Services, CDC; 2015. http:// www.cdc.gov/tb/statistics/reports/2014/default.htm

3. CDC. Tuberculosis screening and treatment technical instructions (TB TIs) using cultures and directly observed therapy (DOT) for panel physicians. Atlanta, GA: US Department of Health and Human Services, CDC; 2015. http://www.cdc.gov/immigrantrefugeehealth/exams/ti/ panel/tuberculosis-panel-technical-instructions.html

4. Bureau of Labor Statistics. Foreign-born workers: labor force characteristics-2014. Washington, DC: US Department of Labor, Bureau of Labor Statistics; 2015. http://www.bls.gov/news.release/pdf/ forbrn.pdf

5. Ghosh S, Moonan PK, Cowan L, Grant J, Kammerer S, Navin TR. Tuberculosis genotyping information management system: enhancing tuberculosis surveillance in the United States. Infect Genet Evol 2012;12:782-8. http://dx.doi.org/10.1016/j.meegid.2011.10.013

6. Mazurek GH, Jereb J, Vernon A, LoBue P, Goldberg S, Castro K. Updated guidelines for using interferon gamma release assays to detect Mycobacterium tuberculosis infection-United States, 2010. MMWR Recomm Rep 2010;59(No. RR-5).

7. Office of Visa Statistics. Nonimmigrant visa statistics. Washington, DC: US Department of State, Bureau of Consular Affairs, Office of Visa Statistics; 2013. https://travel.state.gov/content/visas/en/law-and-policy/ statistics/non-immigrant-visas.html

8. Grieco EM. Length of visit of nonimmigrants departing the United States in 2003. Washington, DC: U.S. Department of Homeland Security, Office of Immigration Statistics; 2005. https://www.dhs.gov/ xlibrary/assets/statistics/publications/LengthVstNonim2003.pdf

9. Davidow AL, Katz D, Ghosh S, et al.; Tuberculosis Epidemiologic Studies Consortium. Preventing infectious pulmonary tuberculosis among foreign-born residents of the United States. Am J Public Health 2015;105:e81-8. http://dx.doi.org/10.2105/AJPH.2015.302662

10. CDC. Antibiotic resistance solutions initiative. Atlanta, GA: US Department of Health and Human Services, CDC; 2015. http://www. cdc.gov/drugresistance/solutions-initiative 